\title{
Disease-Induced Microbial Shifts in Citrus Indicate Microbiome-Derived Responses to Huanglongbing Across the Disease Severity Spectrum
}

\author{
Nichole A. Ginnan, ${ }^{1}$ Tyler Dang, ${ }^{1}$ Sohrab Bodaghi, ${ }^{1}$ Paul M. Ruegger, ${ }^{1}$ Greg McCollum, ${ }^{2}$ Gary England, ${ }^{3}$ Georgios Vidalakis, ${ }^{1}$ \\ James Borneman, ${ }^{1}$ Philippe E. Rolshausen, ${ }^{4}$ and M. Caroline Roper ${ }^{1, \dagger}$ \\ ${ }^{1}$ Department of Microbiology and Plant Pathology, University of California, Riverside, CA 92506, U.S.A. \\ ${ }^{2}$ United States Department of Agriculture-Agricultural Research Service, Fort Pierce, FL 34945, U.S.A. \\ ${ }^{3}$ Agricultural Extension Center, University of Florida, Hastings, FL 32145, U.S.A. \\ ${ }^{4}$ Department of Botany and Plant Sciences, University of California, Riverside, CA 92506, U.S.A.
}

Accepted for publication 11 July 2020.

\section{ABSTRACT}

Plant microbiomes are critical components to plant health and can influence disease outcomes. We provide empirical data describing disease-induced shifts within the citrus microbiome at different levels of huanglongbing (HLB) disease severity. HLB is associated with an invasive phloem-limited bacterium, 'Candidatus Liberibacter asiaticus', that is introduced into the aerial portions of the tree by an insect vector. Disease manifests as aboveground foliar and fruit symptoms and significant root decline belowground. During the early phase of disease, there were depletions of putative keystone taxa in leaves and roots, followed by enrichments of putative beneficial taxa, suggesting a microbially derived immune response involved in plant protection that is ancillary to immune components encoded in the plant's genome. In the late phase of disease, we observed enrichments of parasitic and saprophytic microorganisms, particularly in the roots. The community shifts within the root compartment are emblematic of a disease-induced dysbiosis where pathogens other than ' $\mathrm{Ca}$. $\mathrm{L}$. asiaticus' begin to dominate the community. Furthermore, we define key taxa enriched in trees with a slower rate of disease development, referred to as survivor trees, that are hallmarks of those found in trees in the early phase of disease that may be drivers of the survivor tree phenotype. We propose a disease ecology model that illustrates the relationship between the pathogen, the microbiome, and the host plant that highlights microorganisms that may serve as disease facilitators or antagonists.

Keywords: agriculture, 'Candidatus Liberibacter asiaticus', CLas, holobiont, microbiome, phytobiome, plant pathology, rhizosphere and phyllosphere
${ }^{\dagger}$ Corresponding author: M. C. Roper; mcroper@ucr.edu

N. A. Ginnan and T. Dang contributed similarly to this work.

G. Vidalakis, J. Borneman, and P. E. Rolshausen shared corresponding authorship with M. C. Roper.

Funding: Citrus Research Board Grant Numbers 5300-164 and 6100; California Department of Food and Agriculture Grant Number SCB16056; National Institute of Food and Agriculture Grant Number 2017-70016-26053 and Hatch Projects Grant Numbers 1002710, 1018010, 233883, and 233744; and National Science Foundation Graduate Research Fellowship Program Directorate for Biological Sciences Grant Number NSF DGE-1326120. Any opinions, findings, and conclusions or recommendations expressed in this material are those of the author(s) and do not necessarily reflect the views of the National Science Foundation.

(C) 2020 The American Phytopathological Society
Author contributions: N.A.G. drafted the manuscript; contributed to the sample collection and processing; performed data analyses and interpretation; and conceived, designed, and established the microbiome disease model. T.D. organized sample collection and processing, and performed nucleic acid extractions and qPCR analyses. S.B. contributed to sample collection and processing, and contributed to the high-throughput sequencing (HTS) library construction. P.M.R. constructed and preprocessed HTS libraries and assisted in bioinformatic and statistical analyses. G.M. contributed to sample collection and processing. G.E. identified orchards for sample collection. G.V. coconceived the study and field sites. J.B. coconceived the study and aided in bioinformatic and statistical analyses. P.E.R. coconceived the study, designed and led the field sampling, assisted in data interpretation, and contributed to the writing and editing of the manuscript. M.C.R. coconceived the study, assisted in data interpretation, and contributed to the writing and editing of the manuscript.

*The $\boldsymbol{e}$-Xtra logo stands for "electronic extra" and indicates that supplementary materials and six supplementary tables are published online.

The author(s) declare no conflict of interest. 
A breadth of knowledge about how microbes interact with plants has been derived from studies examining the binary interactions between one pathogen and one plant host. Pathogenic microbes that are introduced into a host must function not only in the context of the eukaryotic host environment but also within the context of the resident microbial community in and around that host (Lamichhane and Venturi 2015; Trivedi et al. 2016; Vandenkoornhuyse et al. 2015; Vorholt 2012). Thus, plant health and disease outcomes can depend on the interactions occurring between the host, its resident microbiome, and the invading pathogen as well as environmental conditions. Plant-associated microbiota play a plethora of roles in plant health that include promoting plant growth, providing tolerance to abiotic stresses, and suppressing or preventing pathogen ingress (Bent and Chanway 1998; Mendes et al. 2011; Ritpitakphong et al. 2016; Rolli et al. 2015; Van der Ent et al. 2009). Members of the microbiome can suppress pathogens through direct competition (nutrient sequestration and antibiosis) or by stimulating plant immunity to resist or tolerate pathogen infections. These interactions are proposed to compose a plant's extended immune system that is auxiliary to plant genome-encoded immune components (Vannier et al. 2019). In contrast, when pathogenic microbes are introduced into a system, the resident microbiota can be susceptible to damaging alterations in community composition as a result of pathogen disturbance. This change in microbiome structure, coupled with the pathogen's production of its own virulence factors, can result in the rapid decline of the plant host (Müller et al. 2016; Schlaeppi and Bulgarelli 2015).

Citrus is a perennial fruit tree crop that is of significant economic, nutritional, and health importance because of the myriad of nutrients, antioxidants, vitamins, minerals, and dietary fiber found in fresh and juiced citrus fruit. Citrus flavors are also classified among the most recognizable and preferred in the world (Baker 1994; Economos and Clay 1999; Timmer et al. 1999). Currently, huanglongbing (HLB), a devastating citrus disease, threatens the sustainability of the citrus industry worldwide and in the United States, particularly in the state of Florida (Babcock 2018; da Graça et al. 2016; Gottwald 2010). In the United States, HLB is associated with an $\alpha$-proteobacterium, 'Candidatus Liberibacter asiaticus', that is vectored by Diaphorina citri (Asian citrus psyllid) (da Graça et al. 2016). ' $C a$. L. asiaticus' is not amenable to axenic culture and has only recently been reported to be successfully cocultured within a microbial consortium, indicating that it is likely highly dependent on cohabitating microbes for growth (Fujiwara et al. 2018). ' $C a$. L. asiaticus' colonizes the phloem tissue of citrus trees and causes ultrastructural changes in the phloem (da Graça et al. 2016; Folimonova and Achor 2010). HLB symptoms include chlorosis ("blotchy mottle" on the leaves, yellow shoots), tree stunting, a major decline in fruit yield, and, ultimately, the death of the tree (da Graça et al. 2016). There is also a marked decline in fruit quality due to the buildup of acidic compounds that leads to a bitter, saltytasting flesh or juice, making it unpalatable (Dagulo et al. 2010). The manifestation of HLB causes an abnormal abundance of starch deposition in leaves and an increase in callose deposition in the phloem sieve elements (Folimonova and Achor 2010; Gonzalez et al. 2011). In addition to the symptoms that manifest in the aerial portions of the tree, root decline also occurs in the subterranean portions of the tree in HLB-affected trees (Graham et al. 2013). Because of the threat HLB poses to citriculture worldwide and the lack of long-term sustainable control measures, there is increased interest in the citrus microbiome and how it relates to HLB (Wang et al. 2015). Several recent studies on the citrus microbiome have provided a foundation of data regarding rhizosphere- or leafassociated microbes of citrus affected by HLB (Blaustein et al. 2017; Sagaram et al. 2009; Trivedi et al. 2010; Xu et al. 2018;
Zhang et al. 2017). Blaustein et al. (2017) hypothesized that bacterial community richness in leaf tissues decreases as HLB symptoms progress and that the relative abundance of Liberibacter spp. was negatively correlated with bacterial community $\alpha$ diversity in leaf tissues. They also predicted negative interactions between Liberibacter spp. and specific bacterial families within the native leaf bacteriome of citrus that may extrapolate to the whole-tree level. These key findings and speculations prompted us to spatially define changes in not only the bacterial communities but also the fungal communities in leaves, their cognate stems, as well as roots using a fine-resolution disease rating scale that parsed trees into five different categories based on their HLB severity. In tandem with this fine-resolution spatial study, we incorporated a temporal disease ecology component to assess longer-term effects of HLB over a 3-year period. Specifically, we investigated trees that developed disease at a slower rate than neighboring trees within the same grove despite the grove being at or near $100 \%$ level of infection with ' $\mathrm{Ca}$. L. asiaticus'. These trees are referred to as survivor trees (Riera et al. 2017; Wang and Gmitter 2014). Considering that citrus trees are clonally propagated on clonal or nucellar (somatic) rootstocks to provide genetically identical trees in a given orchard, we hypothesized that the slow manifestation of HLB symptoms in survivor trees is microbially mediated and derived from the resident citrus microbiota that serves as an additional line of defense against disease, in addition to immune responses emanating from the plant's endogenous defense response.

The dynamics of the citrus microbiome across the disease spectrum revealed specific disease-induced microbial enrichments that occur during early disease onset and during the late phase of disease. We also identified microbial signatures emblematic of keystone taxa that had a stark decline in relative abundance during early disease. Keystone taxa act as community stabilizers in other systems and we speculate that loss of these keystone taxa creates a dysbiosis within the citrus microbiome that allows for a marked increase in sectors of the microbiome that are characteristic of parasitic and saprophytic organisms. Furthermore, this suggests that trees affected by HLB are also coopted by other pathogens and saprophytes. Most interestingly, we phenotyped trees as having decelerated disease progression (survivor trees) at the end of the 3year study and found microbial population signatures enriched in those trees that were emblematic of beneficial microbial taxa. The survivor trees also shared microbial enrichments with trees with low disease severity, suggesting that these microbial populations may be the drivers of the survivor tree phenotype. Based on our findings, we propose a disease ecology model that highlights microbes that represent biomarkers of plant health and disease, and those that are associated with an attenuated disease phenotype that could be developed into potential bioinoculants or biocontrol agents for the HLB-citrus pathosystem.

\section{MATERIALS AND METHODS}

Field sampling of citrus tissues. Leaves, stems and roots from 40 trees were collected in March for three consecutive years (2016, 2017, and 2018) from four different citrus orchards in Florida (Supplementary Table S1). Prior to sampling each year, trees were assessed for HLB severity using a 1-to-5 disease rating (DR) scale, where 1 = vigorous with slight symptoms, $2=$ slight decline, $3=$ moderate decline, $4=$ severe decline, and $5=$ dead or dying, as previously reported (Ginnan et al. 2018; Rouse et al. 2010). We designed our sampling time to coincide with maximum annual ' $\mathrm{Ca}$. L. asiaticus' titers, which typically occurs in March (Blaustein et al. 2017). Each tree was divided into four quadrants (north, south, east, and west) and stems with attached, fully expanded leaves were 
collected from each of the quadrants and pooled. Feeder roots were sampled from two sides of the tree approximately $0.5 \mathrm{~m}$ away from the base of the trunk and sealed in a plastic bag. Gloves were changed and clippers and shovels were sterilized with $30 \%$ household bleach between each tree that was sampled. All samples were immediately placed on ice in a cooler for transit to the laboratory, where they were placed at $4^{\circ} \mathrm{C}$ and processed within $24 \mathrm{~h}$ according to Ginnan et al. (2018). Samples were stored at $-80^{\circ} \mathrm{C}$ and then lyophilized with a benchtop freeze dryer (Labconco FreeZone 4.5L; Labconco, Kansas City, MO, U.S.A.) for 16 to $20 \mathrm{~h}$. The lyophilized tissue was shipped to the University of California Riverside (United States Department of Agriculture permit P526P16-00352) on dry ice.

DNA isolation from plant material. Plant tissue was processed and DNA extractions were performed according to published protocols (Ginnan et al. 2018). Specifically, the samples were not surface sterilized; thus, our microbial next-generation sequencing datasets and analyses included both epiphytes and endophytes. Budwood and leaf samples were cut into 1- to 2-cm-long pieces, and approximately $6 \mathrm{~g}$ of leaves and $10 \mathrm{~g}$ of budwood were placed into $50-\mathrm{ml}$ conical tubes and stored at $-80^{\circ} \mathrm{C}$; root samples were rinsed with autoclaved purified water (Barnstead Mega-Pure System MP-6a; Thermo Fisher Scientific, Waltham, MA, U.S.A.) and approximately $5 \mathrm{~g}$ of rinsed root tissue was placed into $50-\mathrm{ml}$ conical tubes and stored at $-80^{\circ} \mathrm{C}$. Freeze-dried leaves and roots were crushed into small pieces $(<0.5 \mathrm{~cm})$ with sterile stainless-steel spatulas, and $100 \mathrm{mg}$ of freeze-dried tissue was transferred to 2-ml microcentrifuge tubes (Eppendorf Safe-Lock tubes; Eppendorf, Hamburg, Germany) containing a single 4-mm stainless-steel grinding ball (SPEX SamplePrep, Metuchen, NJ, U.S.A.). Approximately $200 \mathrm{mg}$ of whole, freeze-dried budwood was transferred to 5-ml frosted polyethylene vials containing a single $9.5-\mathrm{mm}$ stainless-steel grinding ball (SPEX SamplePrep). Samples were chilled at $-80^{\circ} \mathrm{C}$ for $15 \mathrm{~min}$, then pulverized to a powder using a $2010 \mathrm{Geno} / \mathrm{Grinder}$ (SPEX SamplePrep) at 1,680 rpm for 20 to $30 \mathrm{~s}$, twice. Then, $1 \mathrm{ml}$ of $4 \mathrm{M}$ guanidine thiocyanate was added to the pulverized leaf and root samples. Pulverized budwood was transferred from the 5-ml frosted polyethylene vials to 5-ml microcentrifuge tubes (Eppendorf Safe-Lock tubes); then, $2 \mathrm{ml}$ of $4 \mathrm{M}$ guanidine thiocyanate was added. All samples were incubated at $4^{\circ} \mathrm{C}$ for $15 \mathrm{~min}$ and subsequently centrifuged for $1 \mathrm{~h}$ at $17,500 \times g$. DNA was isolated using the MagMAX-96 DNA Multi-Sample Kit (Thermo Fisher Scientific) with the protocol "4413021ForPlants" on a MagMAX Express-96 Deep Well Magnetic Particle Processor. The final DNA was eluted in $100 \mathrm{ml}$ of DNA elution buffer and the dried DNA was stored at $-20^{\circ} \mathrm{C}$ until utilized for bacterial and fungal Illumina library construction and detection of Phytophthora spp. with quantitative PCR (qPCR). Detection of the Phytophthora genus was performed as previously described (Bilodeau et al. 2014; Miles et al. 2017).

High-throughput sequencing analyses and statistical tests. Illumina bacterial ribosomal RNA (rRNA) internal transcribed spacer (ITS) and fungal rRNA ITS2 libraries were constructed as previously described (Ginnan et al. 2018; Ruegger et al. 2014). Raw sequence reads were uploaded to the NCBI Sequence Read Archive and are accessible under BioProject ID PRJNA590541. We used the UPARSE pipeline for demultiplexing, length trimming, quality filtering, and operational taxonomic unit (OTU) picking using recommended guidelines that were initially described by Edgar (2013) and which have been updated at https://www.drive5.com/ usearch/manual/uparse_pipeline.html (Edgar 2013).

For the bacterial rRNA ITS analyses, after demultiplexing and using the recommended 1.0 expected error threshold, sequences were trimmed to a uniform length of $149 \mathrm{bp}$, which kept nearly $91 \%$ of reads. Sequences were then dereplicated and clustered using the UPARSE-OTU algorithm, which also detects and removes chimeric sequences. An OTU table was then generated using the otutab command. OTUs having nonbacterial DNA were identified and enumerated by performing a local BLAST search of their seed sequences against the nucleotide database. OTUs were removed if any of their highest-scoring BLAST hits contained taxonomic IDs within the citrus family, fungal kingdom, or PhiX. Taxonomic assignments to bacterial OTUs were made by finding the lowest common taxonomic level of the highest BLAST hits, excluding unclassified designations.

For the fungal rRNA ITS2, after demultiplexing and using the recommended 1.0 expected error threshold, sequences were trimmed to a uniform length of $290 \mathrm{bp}$, which kept nearly $77 \%$ of reads. Sequences were then dereplicated and clustered into zero-radius OTUs using the UNOISE3 algorithm (Edgar and Flyvbjerg 2015), which detects and removes chimeric sequences. An OTU table was then generated using the otutab command. Taxonomic assignments to the fungal OTUs were performed using the RDP Classifier version 2.12, trained on the ver7_99_s_10.10.2017 release of the UNITE database, and OTUs having nonfungal assignments were removed.

Preprocessed taxonomically assigned OTU tables were imported into R. Samples with less than 1,000 reads were removed. Nonnormalized reads were used to calculate Shannon index measurements, which accounts for both richness and evenness, with the phyloseq::plot_richness() function (McMurdie and Holmes 2013). A ranked sums analysis of variance statistical test (Kruskal-Wallis), followed by a pairwise Dunn's test with Holm's correction for multiple comparisons, were used to calculate $P$ values.

$\beta$-Diversity analyses were performed using $\mathrm{R}$ packages phyloseq and vegan (McMurdie and Holmes 2013; Oksanen et al. 2007). Preprocessed reads were transformed using total sum scaling (TSS) normalization. Using the ordinate() and plot_ordination() functions, a principal coordinate analysis (PCoA) was done on abundance Jaccard dissimilarity distances, which accounts for presence or absence and abundance of OTUs within each sample. Using the stat_ellipse() function, 95\% confidence ellipses were added to further examine groups. A permutational multivariate analysis of variance (PERMANOVA) statistical test was performed using the vegan::adonis() function. Pairwise PERMANOVA with false discovery rate (FDR) correction was accomplished with RVAideMemoire::pairwise.perm.manova() (Hervé 2015). Core microbiota identifications were performed using microbiome::core() with prevalence set at 0.75 and abundance at $\geq 0.1 \%$ (Lahti et al. 2017).

OTU tables were separated by tissue type and normalized using TSS normalization. Low-read OTUs were filtered out of each specific tissue type OTU table using a 1.0e-5 abundance cutoff. Differentially abundant species were identified from each OTU table. Significance was determined using a Kruskal-Wallis test followed by a pairwise Dunn's test, and FDR correction on the 100 most-abundant species within each tissue type. Nonsignificant species were filtered out and significant results can be found in Supplementary Tables S2 and S3. Using ggplot2 and phyloseq:: subset_taxa() function, the relative abundance of specific species was plotted as boxplots (Ginestet 2011; McMurdie and Holmes 2013).

\section{RESULTS}

Citrus core microbiota and community diversity is driven by multiple factors in areas of high HLB pressure. Our data indicated that orchard characteristics (geographical location, rootstock, scion, and age of tree), year of sampling, and tissue type had a significant effect on the citrus microbiome of trees under high HLB 
disease pressure (Supplementary Fig. S1a and b). We aimed to understand microbial taxa consistent across these variables. For this reason, we separated the dataset by tissue type and selected for taxa with $\geq 0.1 \%$ of the total relative abundance of the microbial community and that were present in at least $75 \%$ of all samples within each tissue type. We defined these as tissue-type-specific core microbiomes, which were present regardless of environment and host factors. $\beta$-Diversity analysis showed that plant tissue (roots, stems, or leaves) accounted for 10.6 and $7.1 \%$ of the variation in the overall bacterial and fungal $(P<0.001$, PERMANOVA (Adonis)) community composition, respectively (Fig. 1A and B). A pairwise PERMANOVA indicated that all binary combinations of tissue type were significantly different, with $P<0.001$ for all bacterial and fungal communities. Furthermore, an $\alpha$-diversity analysis indicated that bacterial root communities have a significantly higher Shannon index than leaves $(P<0.001$, Kruskal-Wallis test, with a pairwise Dunn's test and correcting for multiple comparisons with Holm's method) and stems $(P<0.001$, Kruskal-Wallis test, with a pairwise Dunn's test and correcting for multiple comparisons with Holm's method) (Fig. 1C). Conversely, fungal root communities had a significantly lower Shannon index when compared with leaf $(P<$ 0.001 , Kruskal-Wallis test, with a pairwise Dunn's test and correcting for multiple comparisons with Holm's method) and stem $(P<0.001$, Kruskal-Wallis test, with a pairwise Dunn's test and
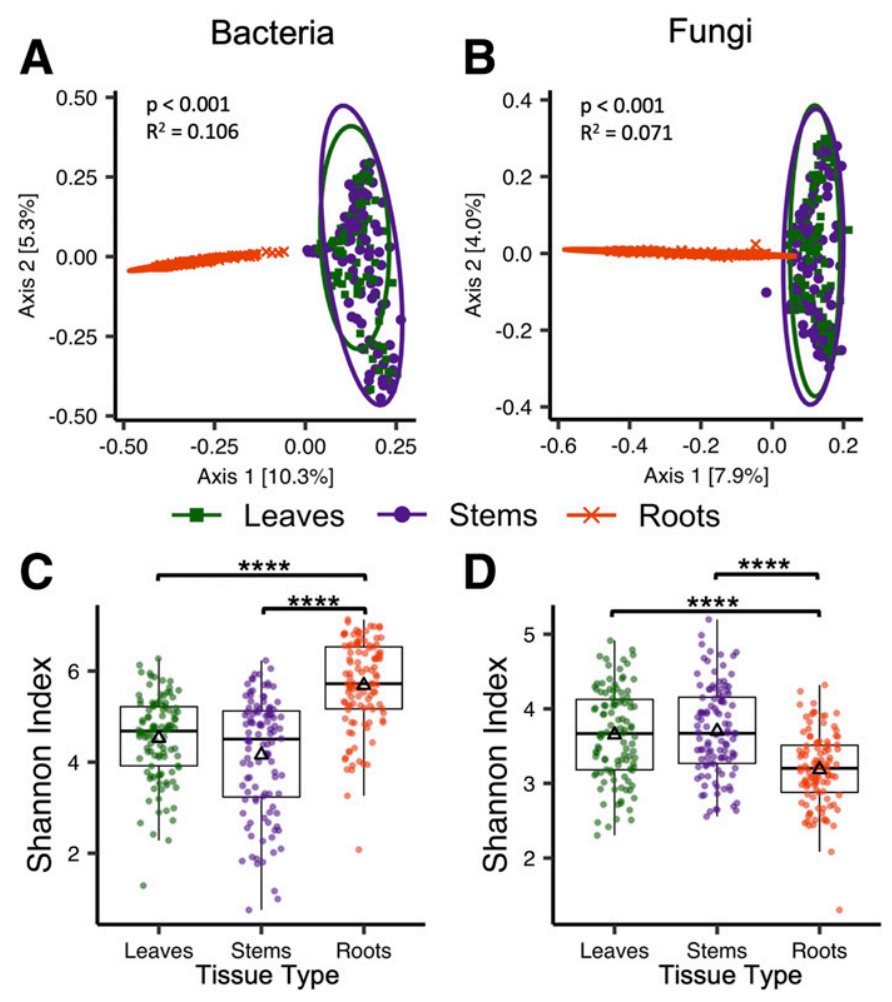

Fig. 1. Plant compartment microbial diversity. $\beta$-Diversity principal coordinate analysis plots of $\mathbf{A}$, bacterial and $\mathbf{B}$, fungal leaf, stem, and root communities. Points are colored by the tissue type and represent a complete community from a single leaf, stem, or root sample. Ellipses represent $95 \%$ confidence intervals. $P$ values and $R^{2}$ values were obtained using a permutational multivariate analysis of variance (Adonis). $\alpha$-Diversity plot of Shannon index measurements of $\mathbf{C}$, bacterial and $\mathbf{D}$, fungal leaf, stem, and root communities. Symbol $\Delta$ represents the mean and asterisks * ${ }^{* *},{ }^{* * *}$, and ${ }^{* \star * *}$ indicate $P \leq 0.05,0.01,0.001$, and 0.0001 , respectively, using a Kruskal-Wallis test, with a pairwise Dunn's test and correcting for multiple comparisons with Holm's method. correcting for multiple comparisons with Holm's method) communities, indicating that fungal root communities were less diverse than leaf and stem fungal communities (Fig. 1D). Leaf, stem, and root core microbiomes were also analyzed separately to identify the ecologically dominant core members for each respective tissue compartment (Table 1). The core microbiomes for these included 11 bacterial genera associated with leaves, 10 associated with stems, and 34 associated with roots. Core mycobiome members included 11 core fungal genera associated with leaves, 8 associated with stems, and 3 associated with roots. The full list of core bacterial and fungal taxa in specific tissue types can be found in Supplementary Table S4.

Disease severity affects community level microbial composition and diversity across the disease spectrum. To investigate diversity and distribution of microbes in leaves, stems, and roots in the context of HLB severity, we initially assessed $\alpha$ diversity for bacterial and fungal communities across the spectrum of the HLB DR scale (DR1 to -5 ) (DR1 $n=16$, DR2 $n=19$, DR3 $n=29$, DR4 $n=31$, and DR5 $n=12$ ) (Fig. 2). The Shannon Index was significantly increased in bacterial communities associated with the stem and root compartments and fungal communities in leaf and stem compartments in trees with higher disease severity as compared with trees with lower disease severity $(P<0.001$, KruskalWallis test, with a pairwise Dunn's test and correcting for multiple comparisons with Holm's method) (Fig. 2A). Interestingly, HLB severity did not affect species richness in leaf bacterial communities or root fungal communities $(P<0.001$, Kruskal-Wallis test, with a pairwise Dunn's test and correcting for multiple comparisons with Holm's method) (Fig. 2A and B).

In addition, $\beta$-diversity analyses showed that the taxonomic structure of both bacterial and fungal communities in all three tissue compartments changed significantly $(P<0.001$, PERMANOVA (Adonis)) with increased disease severity (Fig. 3A and B). Dissimilarity distances were visualized using a PCoA and bacterial and fungal communities clustered by disease status. When examining the two opposite ends of the disease spectrum (DR1 and DR5), bacterial and fungal communities associated with all three tissue compartments in trees with a DR1 were strikingly different in their structural composition from the cognate communities from trees with a DR5 ( $P=0.005$ to 0.0017 , pairwise PERMANOVA), especially in leaf and stem bacterial communities, visually indicated by the nonoverlapping 95\% confidence ellipses (Fig. 3A and B; Supplementary Table S5). Furthermore, the bacterial communities had high homology among trees with the lowest disease status (DR1) in the context of all three tissue compartments. The bacteriomes associated with DR2, DR3, and DR4 trees were more dispersed when compared with DR1 bacteriomes, indicative of a disease-induced dysbiosis (Fig. 3A). When trees were the most severely affected by HLB (DR5), their bacteriome profiles were again more similar to one another (Fig. 3A) yet significantly different than a healthy tree (DR1) bacteriome profile. The mycobiomes associated with leaves and stems showed decreased variability in composition among trees associated with increased disease severity (Fig. 3B).

To determine which taxa drove the shifts in $\alpha$ - and $\beta$-diversity metrics, we initially examined the community shifts at the phyla level. We found significant changes in relative abundance of 9 bacterial phyla associated with leaves, 15 associated with stems, and 10 associated with roots when disease rating increased (Fig. 4A). Additionally, we found significant shifts in the relative abundances of three fungal phyla associated with leaves, four associated with stems, and six associated with roots (Fig. 4B). Notably, the three most abundant bacterial phyla-Proteobacteria, Bacteroidetes, and Actinobacteria - displayed particularly significant 
TABLE 1

Dominant core citrus-associated bacterial and fungal genera in trees under high huanglongbing pressure

\begin{tabular}{|c|c|c|}
\hline Plant compartment and community & Dominant core genera & Relative abundance (\%) \\
\hline Leaf bacteriome & Methylobacterium & 13.1 \\
\hline Leaf bacteriome & Hymenobacter & 11.8 \\
\hline Leaf bacteriome & Streptomyces & 2.6 \\
\hline Leaf bacteriome & Sphingomonas & 1.7 \\
\hline Stem bacteriome & Methylobacterium & 7.3 \\
\hline Stem bacteriome & Hymenobacter & 6.1 \\
\hline Stem bacteriome & Streptomyces & 4.2 \\
\hline Root bacteriome & Streptomyces & 35.4 \\
\hline Root bacteriome & Pseudonocardia & 3.2 \\
\hline Leaf mycobiome & Cladosporium & 21.2 \\
\hline Leaf mycobiome & Symmetrospora & 7.5 \\
\hline Leaf mycobiome & Hannaella & 4.1 \\
\hline Leaf mycobiome & Alternaria & 3.0 \\
\hline Leaf mycobiome & Cryptococcus & 2.1 \\
\hline Stem mycobiome & Cladosporium & 16.8 \\
\hline Stem mycobiome & Symmetrospora & 6.5 \\
\hline Stem mycobiome & Hannaella & 3.9 \\
\hline
\end{tabular}

A



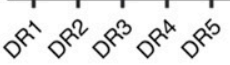

Bacteria

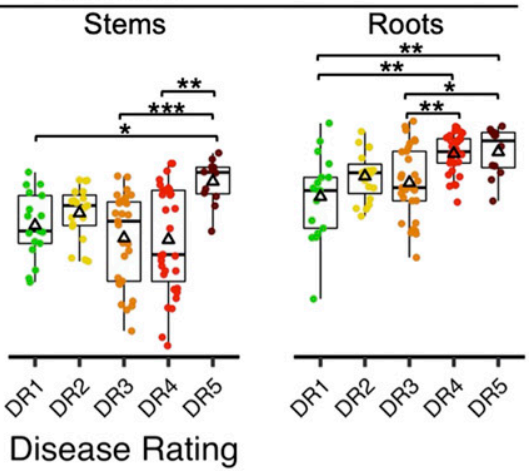

B

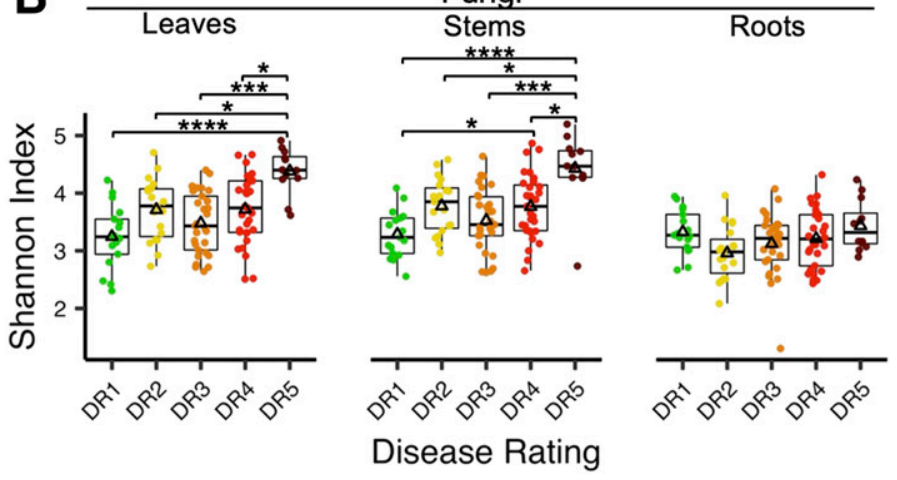

Fig. 2. Disease severity affects community diversity and species richness. $\alpha$-Diversity plot of Shannon index measurements of $\mathbf{A}$, bacterial and $\mathbf{B}$, fungal leaf, stem, and root communities grouped by disease rating (DR) on a scale of DR1 to DR5. Symbol $\Delta$ represent the mean and asterisks *, ${ }^{* *}$, ${ }^{* * *}$, and ${ }^{* \star * \star}$ indicate $P \leq 0.05,0.01,0.001$, and 0.0001 , respectively, using a Kruskal-Wallis test, with a pairwise Dunn's test and correcting for multiple comparisons with Holm's method. 
shifts in relative abundance in the leaf $(P=0.0022,0.0238$, and 0.0247 , respectively; Kruskal-Wallis test $)$ and stem $(P=0.0006$, 0.0366 , and 0.0047 , respectively; Kruskal-Wallis test) tissues when disease severity increased (Fig. 4C). The top three fungal phylaAscomycota, Glomeromycota, and Basidiomycota—had significant changes in relative abundance in the root tissue $(P=0.0123,0.0117$, and 0.0017 , respectively; Kruskal-Wallis test) when disease severity increased (Fig. 4D). A list of all phyla with significant shifts in each tissue type can be found in Supplementary Table S6.

Microbial signatures associated with early and late phases of disease. We mined the dataset for taxa that exhibited distinct patterns of enrichment or reduction in relative abundance across the disease spectrum (Figs. 5 and 6). The bacterial rRNA ITS region was analyzed instead of the 16S rRNA gene because it can provide increased taxonomic resolution (Deyett et al. 2017; Ruegger et al. 2014; Schanche et al. 2015). Fungal ITS2 regions enabled us to assign taxonomy to the fungal microbial enrichments. As expected, the relative abundance of ' $C a$. L. asiaticus' was increasingly enriched in leaf and stem tissue compartments with increased HLB severity rating (Fig. 6A), which corroborates findings by Blaustein et al. (2017). Absolute abundance of ' $\mathrm{Ca}$. L. asiaticus', determined by qPCR, also increased as HLB severity rating increased in leaves and stems. Interestingly, ' $C a$. L. asiaticus' had a sharp decline in relative abundance in leaves and stems of severely affected trees (DR5) (Fig. 6A) but did not exhibit a significant decrease in absolute abundance in leaves or stems. ' $\mathrm{Ca}$. L. asiaticus' relative abundance remained stable across the disease spectrum in roots but significantly decreased in absolute abundance in DR5 trees when compared with the DR1 trees on the other end of the disease spectrum (Supplementary Fig. S2).

We then categorized microbial enrichment patterns for other dominant microbes in trees with specific disease phenotypes. Several bacterial and fungal taxa were enriched in trees with low to moderate levels of disease (DR1 to -3). We define these as microbial disease signatures of early disease onset (Fig. 5A to C). The fungal populations (Exophiala sp. in roots and Aureobasidium sp. in leaves) and the bacterial populations (Methylobacterium spp. in leaves and Lactobacillus spp. in roots) were enriched in trees in the early to moderate stages of disease (DR2 and DR3) but dropped significantly in relative abundance in highly HLB-symptomatic

A

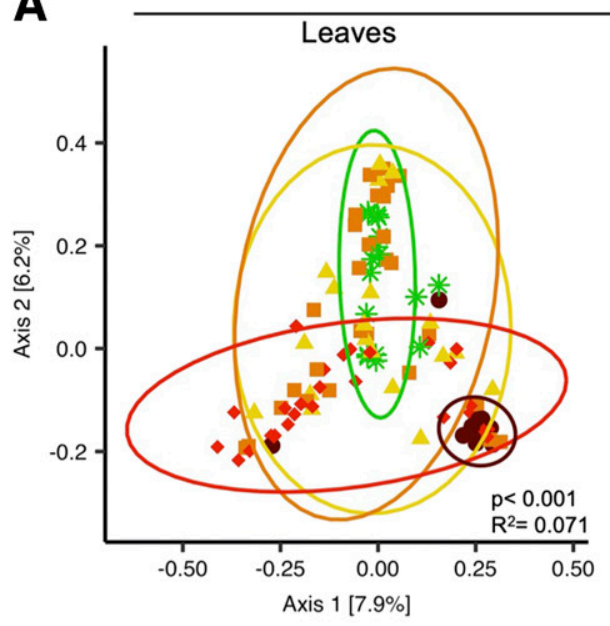

Bacteria
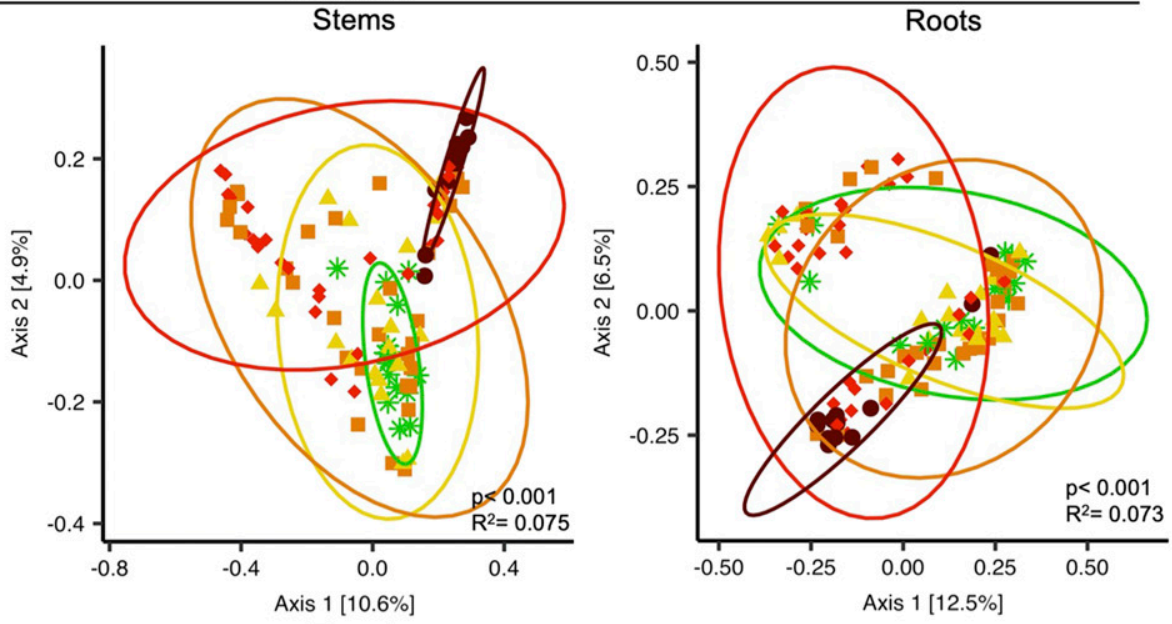

B

Fungi



* DR1
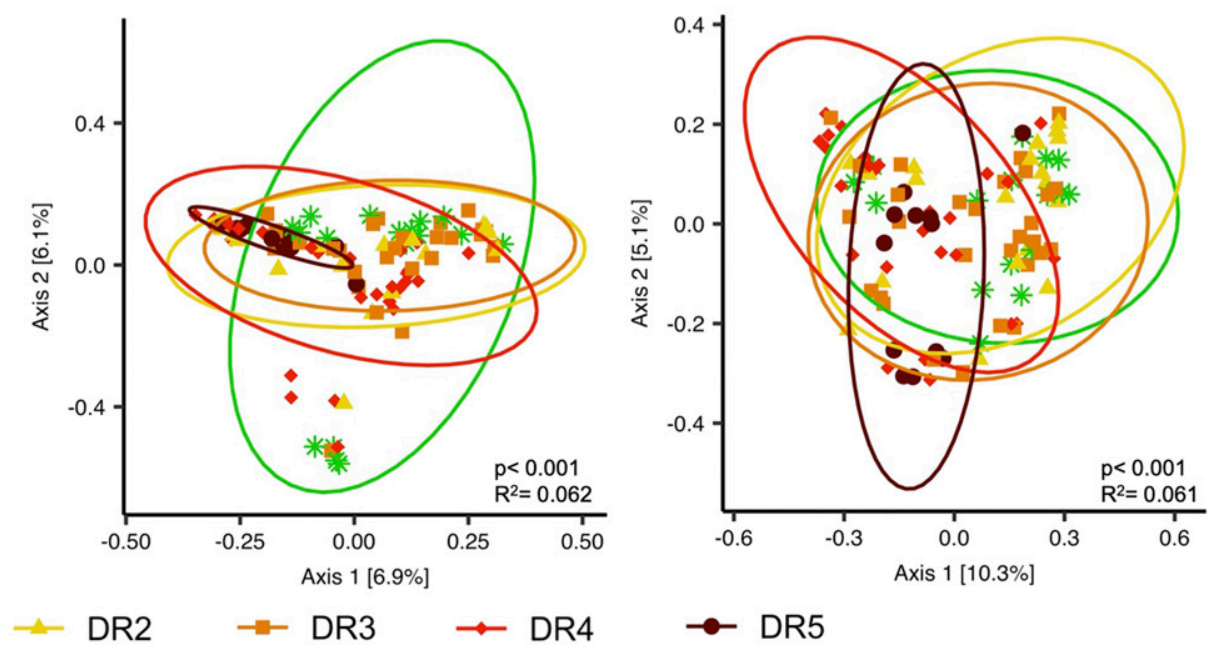

Fig. 3. Disease severity affects $\beta$ diversity. $\beta$-Diversity principal coordinate analysis plots of $\mathbf{A}$, bacterial and $\mathbf{B}$, fungal leaf, stem, and root communities. Points are colored by the disease rating (DR on a scale of 1 to 5 ) of the tree and represent a complete community from a single leaf, stem, or root sample. Ellipses represent $95 \%$ confidence intervals. $P$ values and $R^{2}$ values were obtained using a permutational multivariate analysis of variance (Adonis). 
A

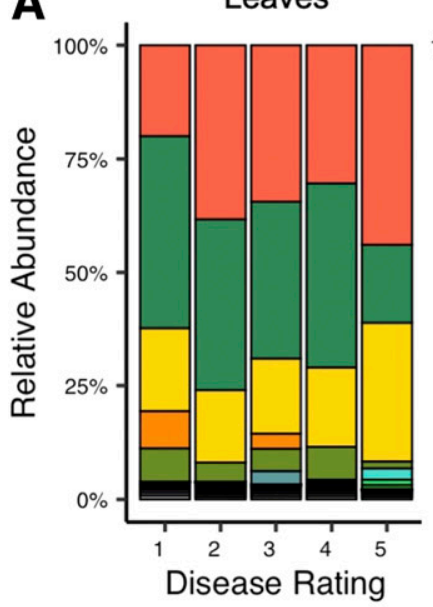

B

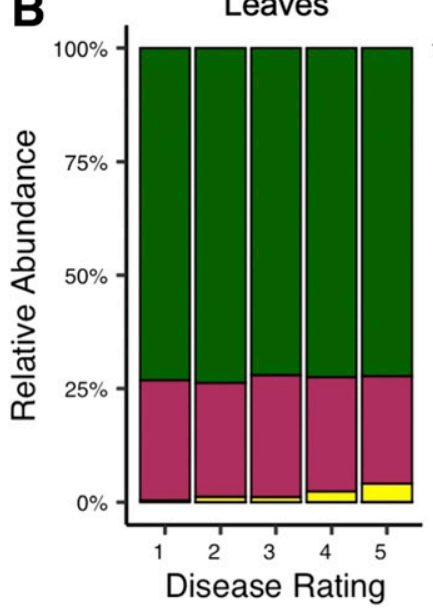

Stems

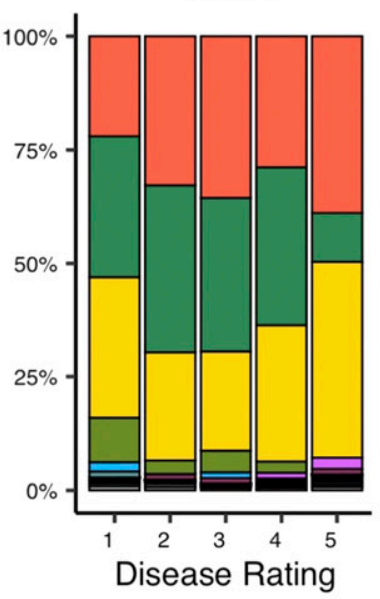

Stems

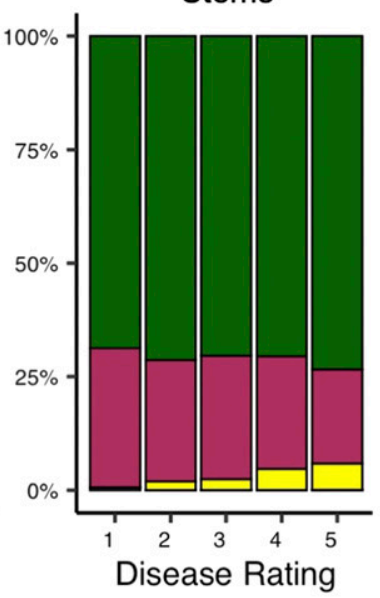

C

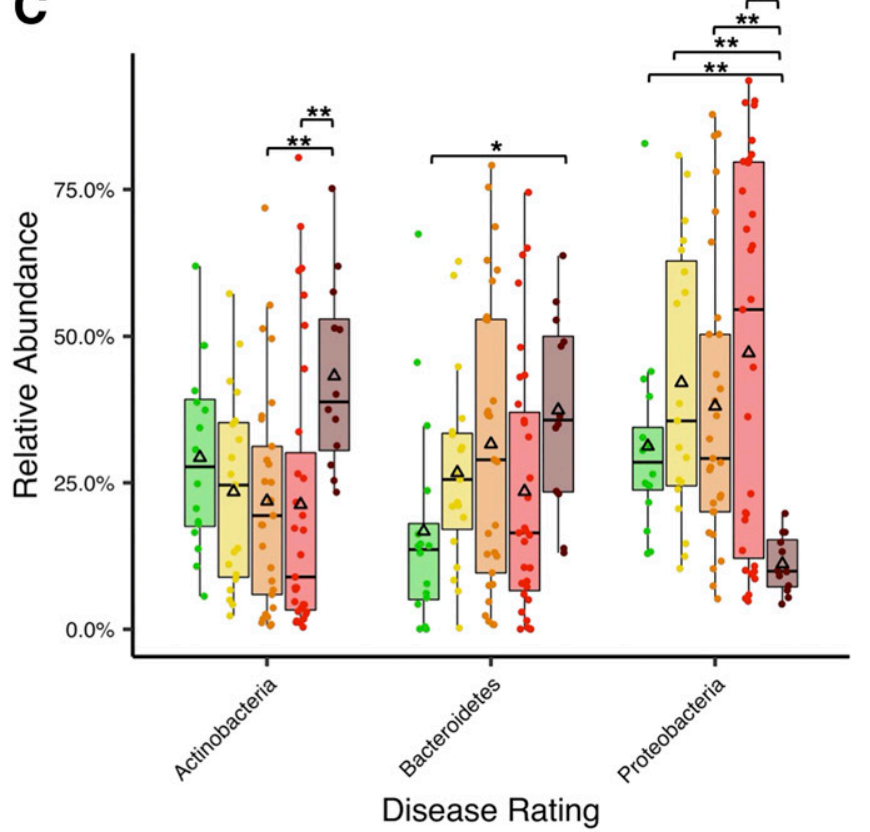

Roots



Roots

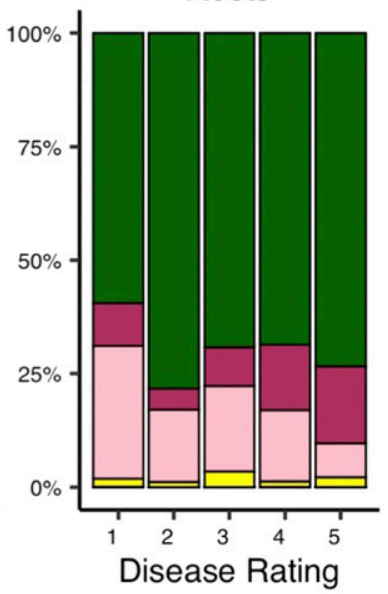

D

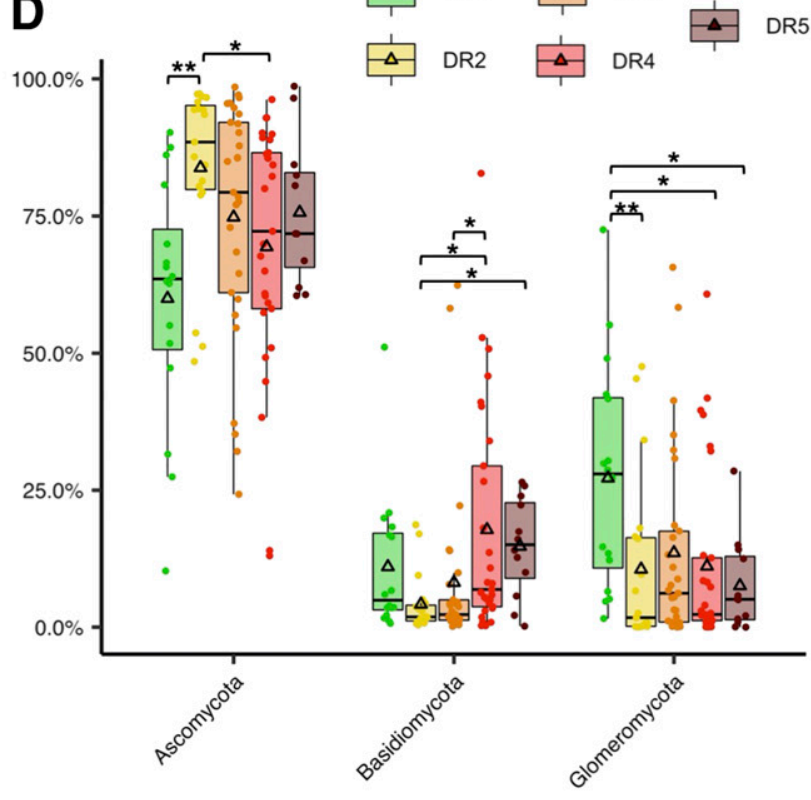

Disease Rating

Fungal Phyla

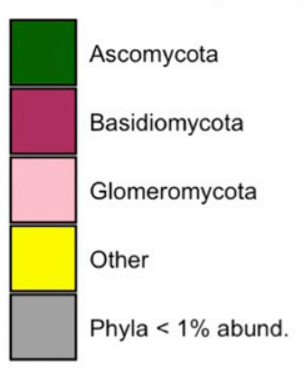

\section{Disease Rating}

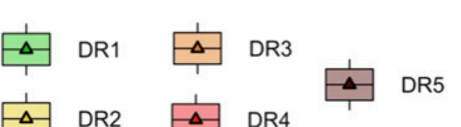

Fig. 4. Disease severity causes shifts in relative abundance and $\alpha$ diversity at the phyla level. Stacked bar plots display A, bacterial and B, fungal community profiles across disease ratings (DR) at the phyla level. The relative abundance across DR for the top three most abundant $\mathbf{C}$, bacterial stem phyla and $\mathbf{D}$, fungal root phyla are presented with boxplots. Symbol $\Delta$ represents the mean relative abundance and asterisks ${ }^{*},{ }^{\star *},{ }^{\star \star \star}$, and ${ }^{* \star \star \star}$ indicate $P \leq 0.05,0.01,0.001$, and 0.0001 , respectively, using a Kruskal-Wallis test, with a pairwise Dunn's test and correcting for multiple comparisons with false discovery rate correction. 
trees (DR4 and DR5) (Fig. 5A and B). Two fungi found in leaf tissues (Camptophora sp. and Acrodontium sp.) were enriched in trees with very low levels of disease (DR1) but had a sharp decline in relative abundance in severely diseased trees (Fig. 5C). Additional



\section{B Biological Control Agents}

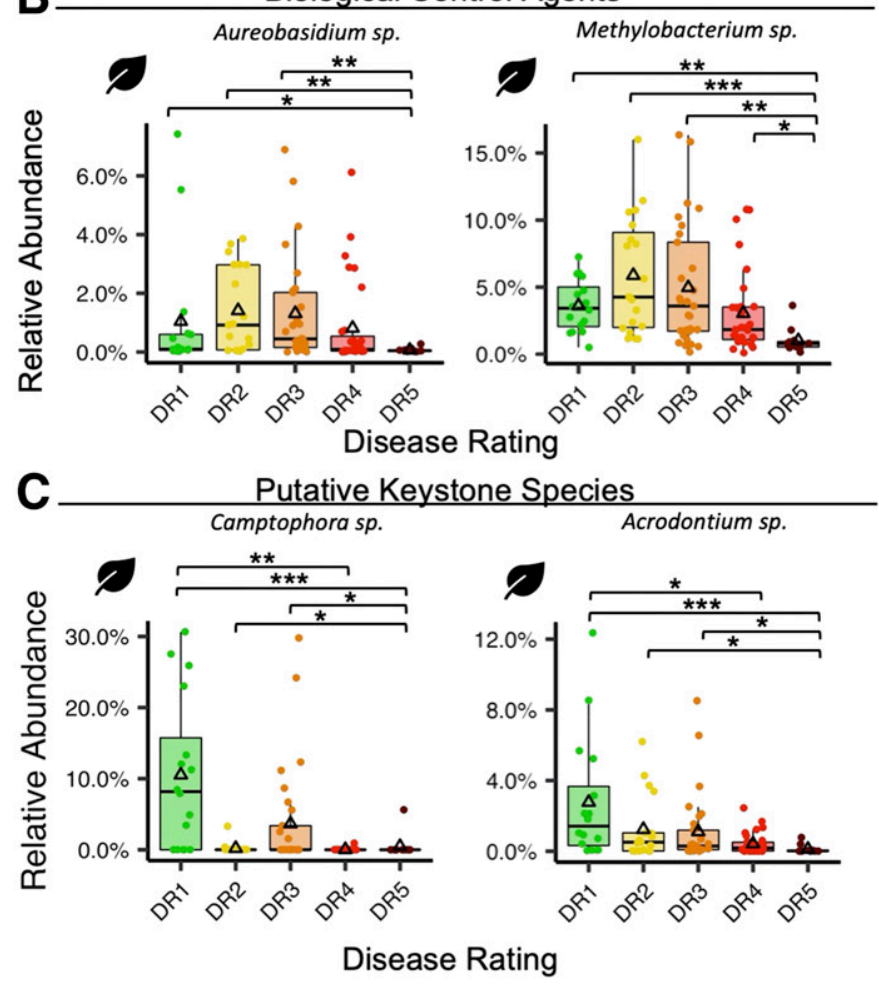

Fig. 5. Microbial signatures during early disease onset for A, plant-growthpromotion isolates; $\mathbf{B}$, biological control agents; and $\mathbf{C}$, putative keystone species. Specific bacterial and fungal populations displayed early enrichment response patterns as the disease rating (DR on a scale of 1 to 5 ) increased. Leaf, stem, and root icons represent population abundances from those specific tissues. Symbol $\Delta$ represents the mean relative abundance and asterisks *, ${ }^{* *}$, ${ }^{* \star *}$, and ${ }^{* \star \star *}$ indicate $P \leq 0.05,0.01,0.001$, and 0.0001 , respectively, using a Kruskal-Wallis test, with a pairwise Dunn's test and correcting for multiple comparisons with false discovery rate correction. populations with these enrichment patterns are shown in Supplementary Figure S3 and listed in Supplementary Table S2.

Furthermore, we identified taxa with enrichment patterns that we classified as late disease microbial signatures. There were notable increases in the relative abundance of putative fungal pathogens in the roots of severely HLB-affected trees (Fig. 6B; Supplementary Table S2). This includes Fusarium spp. and Gibberella spp. Because the fungal ITS2 high-throughput sequencing primers used in this study did not target oomycete species, we performed qPCR using Phytophthora genus-specific primers. Results showed that trees had higher incidences of Phytophthora spp. associated with their roots when they had higher HLB disease ratings (Fig. 6B). Additionally, known fungal saprophytes such as Coprinopsis sp. and Cyphellophora sp. were enriched in roots and stems, respectively, in severely diseased trees with a rating of DR5 (Hoegger et al. 2004; Réblová et al. 2013) (Fig. 6B; Supplementary Fig. S3a). Two Streptomyces spp. in roots, which produce multiple antimicrobials, were enriched during late stages of disease (DR4) and then declined in relative abundance (Fig. 6C) (Liu et al. 2018). Several additional bacterial and fungal populations showed significant enrichment or depletion patterns during early disease onset or late disease; however, little is known about their biological functions. These additional populations with these enrichment or depletion patterns can be found in Supplementary Table S2.

Our findings also indicated that several microbial populations were stable in relative abundance across the disease spectrum. These included the bacteria Bacillus sp., Niastella sp., Lentzea sp., Gemmatimonas uncultured, and Rhodococcus sp. from roots, and Sphingomonas spp. from stems (Supplementary Fig. S4a to f).

Microbial signatures associated with a slower rate of disease development. We also examined the rate of disease development over the 3-year study. We categorized trees with a decelerated disease progression (DDP) phenotype and termed these survivor trees $(n=18)$. Trees were considered to have a DDP when two criteria were met: (i) a tree had $\leq 1$ disease severity rating increase over the 3-year time period and (ii) the disease rating was $<$ DR4 over the course of the 3-year time period. Trees whose disease phenotype did not meet those criteria were considered as nonsurvivor $(n=89)$ trees and represented trees with a typical rate of HLB development. Survivor and nonsurvivor trees had no significant differences in ' $\mathrm{Ca}$. L. asiaticus' relative abundance in leaves, stems, or roots (Supplementary Fig. S5). However, we did observe significant enrichments of other sectors of the microbial community in survivor trees. Specifically, the bacterium Lactobacillus sp. was significantly enriched in roots of survivor trees (Fig. 7A). Lactobacillus spp. have plant growth-promoting properties (Giassi et al. 2016; Quattrini et al. 2018). Two fungi with the highest species alignment to Exophiala sp. (roots) and Aureobasidium sp. (leaves) were enriched in survivor trees (Fig. 7A). Aureobasidium pullulans is a biocontrol agent in citrus and other plant systems (Klein and Kupper 2018; Wang et al. 2018).

The microbial populations enriched in nonsurvivor trees included several Streptomyces spp. (Fig. 7B). A comprehensive list of bacteria and fungi significantly enriched in survivor and nonsurvivor trees appears in Supplementary Table S3.

\section{DISCUSSION}

Several models regarding the effects of exogenous disturbances on environmental microbiomes have been proposed (Allison and Martiny 2008). Although these models were primarily built around the effects of abiotic disturbances on microbiome function, they are also suitable for defining effects following the introduction of an invasive biotic pathogen to a plant microbiome system such as ' $\mathrm{Ca}$. 
L. asiaticus' in citrus. The first model predicts that microbiomes can exhibit resilience following a disturbance and can rebound over time back to the original community structure (Allison and Martiny 2008). Over 3 years, our study indicated that the microbiomes of trees undergoing rapid HLB progression lacked resilience under pathogen or disease stress and were significantly disrupted. A second model proposes that microbiomes can exhibit resistance to disturbances and experience no significant shifts in structure or diversity as a result of a particular disturbance (Allison and Martiny 2008). We found that the overall composition of the citrus microbiome was not resistant to ' $\mathrm{Ca}$. L. asiaticus' disturbance and was, in fact, highly susceptible to this disturbance, which causes compositional alterations that are not restored. Overall, at the whole-community level, we observed significant shifts in bacterial and fungal community composition in all three tissues across the disease spectrum that could be detected at broad taxonomic levels (phylum) as well as down to narrow taxonomic levels (genus and putative species).

When examining the communities at the phyla level, the bacterial stem communities had the largest number of phyla that underwent significant changes in relative abundance that corresponded with increased disease severity. Specifically, Proteobacteria, Bacteroidetes, and Actinobacteria were at increased levels in trees that were more severely HLB symptomatic. Not surprisingly, the increase in Proteobacteria was driven largely by an increase in ' $\mathrm{Ca}$. L. asiaticus'. Considering the dominance of ' $\mathrm{Ca}$. L. asiaticus' in the stem communities, it likely appropriates resources within that tissue compartment, which we speculate influences the other bacterial and

A

Candidatus Liberibacter sp.

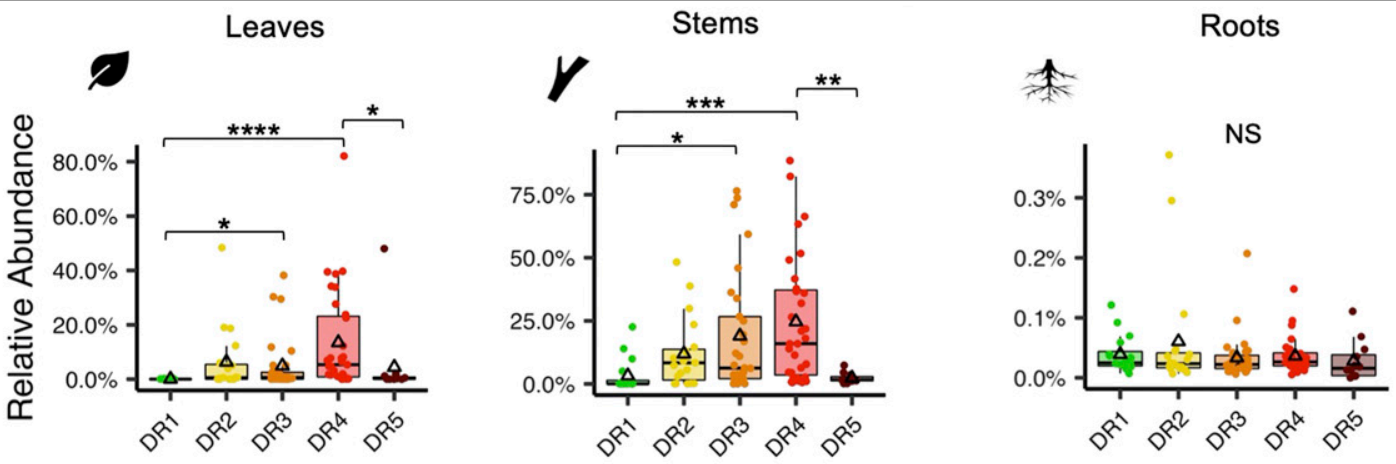

B

Plant Pathogens and Saprophyte
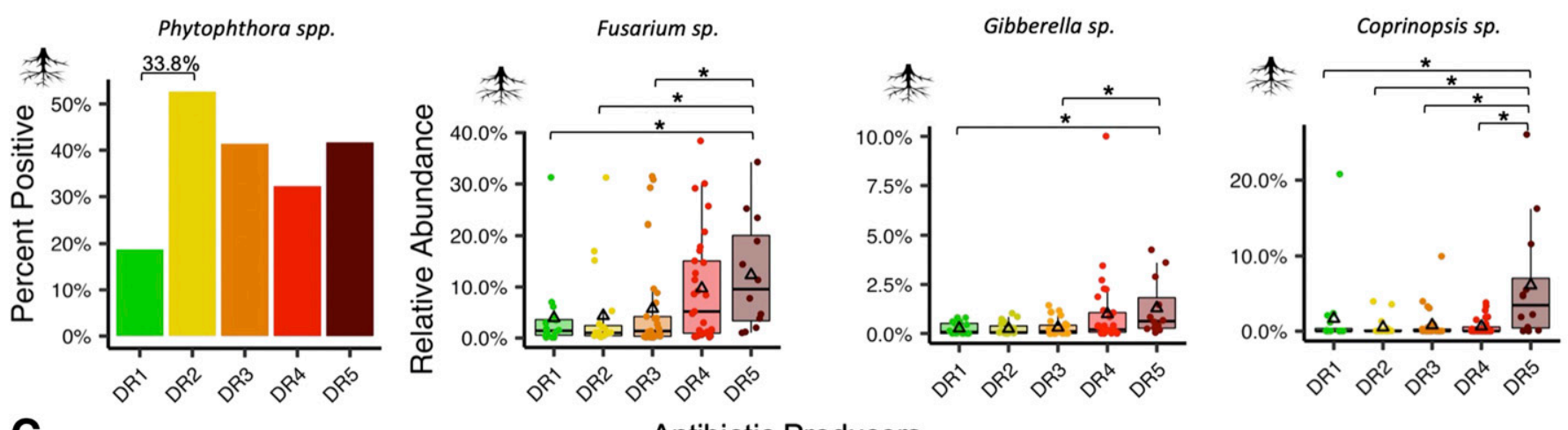

C

Antibiotic Producers

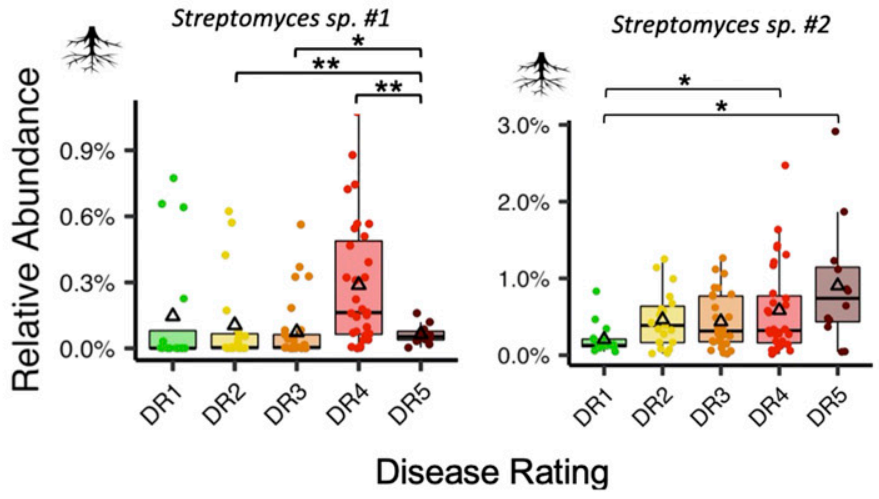

Fig. 6. Microbial signatures of late disease stages. 'Candidatus Liberibacter asiaticus' and other microbial populations that are enriched or depleted in late stages of disease. 'Ca. L. asiaticus' relative abundance in A, three plant compartments; B, pathogens and saprophytes; and C, symbionts. DR = disease rating on a scale of 1 to 5 . Leaf, stem, and root icons represent population abundances from those specific tissues. Symbol $\Delta$ represents the mean relative abundance; asterisks ${ }^{*},{ }^{* *},{ }^{* * *}$, and ${ }^{* * * *}$ indicate $P \leq 0.05,0.01,0.001$, and 0.0001 , respectively; and NS indicates no significant differences using a Kruskal-Wallis test, with a pairwise Dunn's test and correcting for multiple comparisons with false discovery rate correction. 
fungal phyla because ' $\mathrm{Ca}$. L. asiaticus' has fundamentally disrupted and dominated the stem community. There was a significant increase in relative abundance in Actinobacteria when ' $\mathrm{Ca}$. L. asiaticus' increased in relative abundance, which was largely represented by Streptomyces spp. Interestingly, $\alpha$ diversity of root fungal communities was stable across the disease spectrum but did exhibit significant changes in $\beta$ diversity. This illustrates that the root tissue supports a similar number of species in trees with increasing degrees of symptomology but the composition of those communities changes dramatically in trees with severe HLB symptoms. Notably, there was a sharp decrease in relative abundance in the fungal phylum Glomeromycota in the roots of diseased trees. This phylum contains many species known to have beneficial mycorrhizal symbiotic associations with host plant roots that are critical for nutrient and water uptake and contribute to overall root and plant vigor (Willis et al. 2013). In conjunction with this decrease in Glomeromycota, there was a significant increase in the fungal genera Fusarium and Gibberella in roots of trees with significant HLB symptomology. Fusarium oxysporum is associated with citrus dry root rot (Yaseen and D'Onghia 2012) and Gibberella intricans is a Fusarium anamorph that is also known to be a plant pathogen (Lazarotto et al. 2014; Lu et al. 2014). We speculate that the decline in potentially beneficial mycorrhizal fungi coupled with the increase in potential Fusarium or Gibberella fungal parasites is a major contributor to the root decline observed in HLB trees. Previous work indicated that Phytophthora spp. were associated with root decline in HLB-affected trees and our data supported this evidence (Graham et al. 2013). We also observed an increase in the saprophytic organisms Coprinopsis sp. and Cyphellophora sp. in

\section{A}

Enriched in Survivor Trees
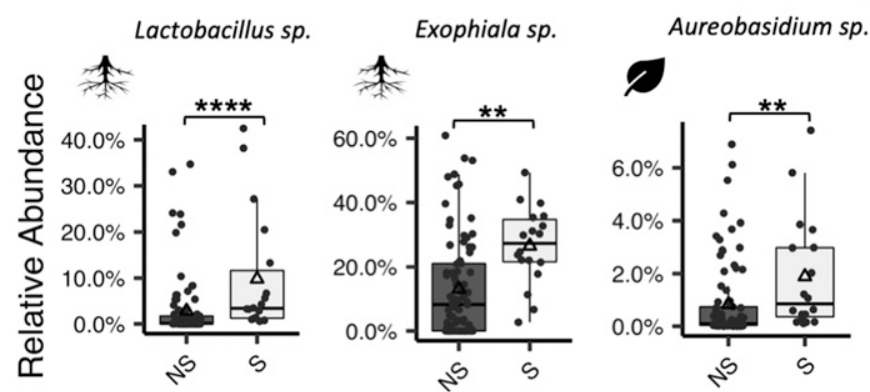

Phenotype

B

Enriched in Non-Survivor Trees
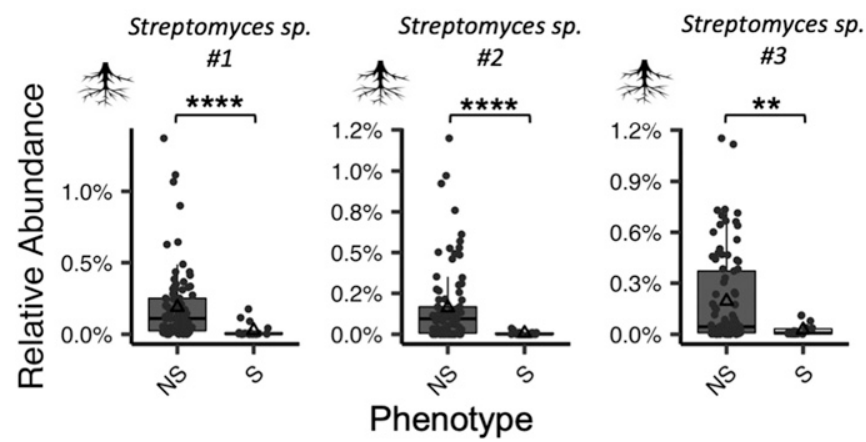

Fig. 7. Bacterial and fungal populations that are significantly enriched in A, survivor (S) or B, nonsurvivor (NS) trees. Leaf, stem, and root icons represent population abundances from those specific tissues. Symbol $\Delta$ represents the mean relative abundance and asterisks ${ }^{*},{ }^{* *},{ }^{* \star *}$, and ${ }^{\star \star \star *}$ indicate $P \leq 0.05,0.01,0.001$, and 0.0001 , respectively using a Kruskal-Wallis test, with a pairwise Dunn's test and correcting for multiple comparisons with false discovery rate correction. the roots of the most symptomatic trees. Together, these data support the idea that a tree's decline is due not just to a ' $\mathrm{Ca}$. L. asiaticus' increase in the aerial portions of the tree but also to large sectors of the root community shifting toward microbes that engage in pathogenic and saprophytic relationships with the host.

We identified microbial enrichments that were induced during early disease onset, suggesting that these represent a sector of the microbiome that is a microbiome-mediated response to pathogen invasion or the physiological parameters surrounding disease onset, as previously observed in other pathosystems (Deyett and Rolshausen 2019). Specifically, the bacterial genera Methylobacterium and Hymenobacter were enriched in leaf communities of trees with low levels of disease (DR1 and -2) and were significantly reduced in relative abundances in the late phase of disease (DR4 and -5). Methylobacterium spp. are known beneficial plant microbes and several species have been tested for their biocontrol properties (Kim et al. 2010; Madhaiyan et al. 2015; Tani et al. 2015). The Hymenobacter genus contains several species that promote plant growth (Dimitrijević et al. 2018). Blaustein et al. (2017) also identified Methylobacterium and Hymenobacter as two genera that were enriched in leaf tissue of asymptomatic citrus trees sampled in an area under HLB disease pressure. This suggests that these two bacteria are emblematic of a healthy citrus leaf microbiome under Florida conditions and that, as trees become more symptomatic, these bacteria are either outcompeted by other microbiota or the diseased leaf environment becomes resource limited and causes a decline in these two bacterial populations. The yeast fungus Aureobasidium sp. was also enriched in leaves of trees with early disease onset and is a common member of native microbiota associated with perennial tree and vine crops. A. pullulans has been developed into an effective biocontrol agent against epiphytic fruit diseases such as Botrytis bunch rot and sour rot of citrus, among others (Klein and Kupper 2018; Wang et al. 2018). The fungus Exophiala sp. was highly enriched in root communities of trees with a low disease state and represented as much as $60.9 \%$ of the relative abundance of the fungal root community during early disease. Xu et al. (2018) identified Exophiala pisciphila as a core member of the global citrus rhizosphere microbiome $(\mathrm{Xu}$ et al. 2018). Heavy-metal-resistant strains of this fungus can protect maize plants from stress caused by excess cadmium and zinc, by partitioning these heavy metals into the fungal cell walls in a seemingly beneficial capacity (Wang et al. 2016). However, when plants were not challenged with heavy metal stress, this same E. pisciphila strain engaged in a parasitic relationship and decreased maize root biomass, suggesting that it is a conditional beneficial microorganism under specific selective conditions. Whether E. pisciphila oscillates between beneficial or parasitic relationships with citrus in the root environment is unclear but warrants further investigation because this fungus represents such a large sector of the citrus root mycobiome.

We also observed a compelling trend in microbial populations during the late phase of disease where several species of Actinobacteria, including those belonging to the bacterial genus Streptomyces, were enriched in DR4 to -5 trees. Streptomycetes are known prolific producers of antibiotics, with streptomycin being an archetypal example of an antibiotic being produced by a Streptomyces sp. (Wang et al. 2016). Interestingly, Streptomycetes engage in antagonistic interactions with Fusarium spp. in the soil (Essarioui et al. 2017, 2019) and competition between these two groups of microbes is largely driven by nutrient resource competition. One can speculate that root decline associated with HLB provides the perfect staging ground for competitive interactions between Streptomyces and Fusarium spp. based on the observed increases in relative abundances of Fusarium spp. in conjunction with an 
increase in relative abundance and species richness of Streptomycetes in highly symptomatic trees. Simultaneously, ' $\mathrm{C}$. L. asiaticus' has a sharp decline in relative abundance in both aerial tissue types in trees rating a DR5 and Streptomyces spp. were also highly abundant in stems tissues in these same trees. Carrión et al. (2019) identified disease-induced sectors of the sugar beet bacterial microbiome in plants infected with the fungal pathogen Rhizoctonia solani that effectively reduced pathogen titer and overall disease severity following pathogen ingress. The significant decrease in both relative and absolute abundance of ' $\mathrm{Ca}$. L. asiaticus' we observed in the late phase of infection in roots may simply be due to a restriction of resources available in a severely diseased tree or degradation of ' $\mathrm{Ca}$. L. asiaticus' DNA. However, it is tempting to conjecture that disease-induced enrichment of Streptomyces in the root tissues is a microbiome-driven immune response that has a suppressive effect on ' $\mathrm{Ca}$. L. asiaticus' in the roots. However, in the case of HLB, this disease-induced enrichment of Streptomyces in the roots is chronologically out of phase with ' $\mathrm{Ca}$. L. asiaticus' colonization and is insufficient to alleviate HLB symptoms in plants.

Additionally, we temporally tracked the rate of disease progression in all trees included in this study and analyzed the data based on the rate at which disease developed in trees that had a slower rate of disease progression over the 3 years and were classified as having the DDP phenotype. Interestingly, specific microbial populations that were significantly enriched in DDP trees had microbial signatures that were similar to those trees that were enriched in the mild to early disease onset category (DR1 to -2) regardless of whether the DDP tree had reached a disease rating higher than DR1 to -2 . These included Aureobasidium, a genus that contains a known biocontrol agent, and Exophiala sp. The functional role of these two fungi in citrus biology is unknown but the high relative abundance of $E$. pisciphila in relatively healthy trees and its enrichment in survivor trees warrants further studies on its role in plant health and disease suppression. The bacterium Lactobacillus sp. was highly enriched in roots of DDP trees. Lactobacillus spp. are known to promote plant growth, especially under stress, and are associated with numerous bioactive metabolites such as 3-phenyllactic acid and 4-hydroxyphenylactic acid, which have broad-spectrum antimicrobial activity (Berthelot et al. 2016; Hussein and Joo 2018; Ignatova et al. 2015; Lavermicocca et al. 2003; Quattrini et al. 2018; Valerio et al. 2004; Wang et al. 2016). Root-associated Lactobacillus spp. can increase both root and shoot biomass of citrus rootstocks, promote root length, and decrease leaf tearing of turfgrass (Baffoni et al. 2012; Giassi et al. 2016). Collectively, because the enrichment of these species is connected to the survivor tree phenotype, we speculate that they may decelerate HLB development by suppressing ' $\mathrm{C}$. L. asiaticus' or alleviating symptoms by an unknown mechanism.

In addition to the phylum Glomeromycota, we also identified other taxa that were in high relative abundance in the healthy trees in the study but significantly decreased beginning at early disease onset. These include Camptophora sp. and Acrodontium sp. and may be holistic indicators of tree health. We propose a model (Fig. 8) where pathogen disturbance causes a destabilization in microbiome complexity that leads to a decrease in putative keystone species that act as community stabilizers, such as Camptophora sp. and Acrodontium sp. We hypothesize that the increase in ' $\mathrm{Ca}$. L. asiaticus', coupled with the observed loss of critical keystone species, initiates a disease-induced dysbiosis in the tree's microbiome. This

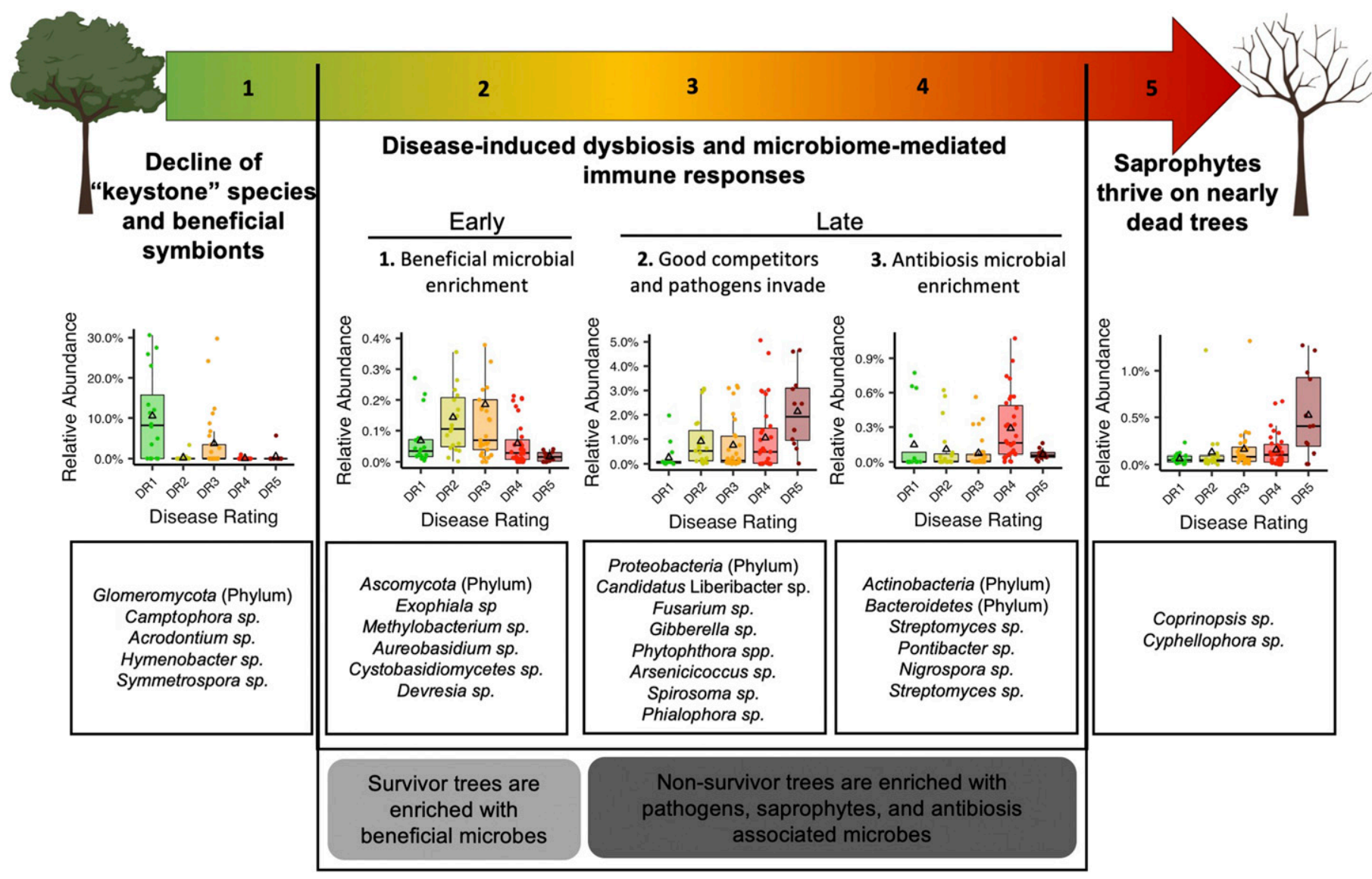

Fig. 8. Huanglongbing microbiome disease model. 
dysbiosis triggers an initial enrichment of known beneficial microbes as part of an early microbiome-initiated immune response in an effort to dampen the proliferation of the invading pathogen within the microbial community. Interestingly, trees with slower rates of disease (DDP survivor trees) are enriched in these beneficial microbes, supporting our hypothesis that these taxa play a crucial role in maintaining plant health and could be indicative of an adaptive beneficial response to disease-induced dysbiosis. In the late phase of disease, there was also secondary disease-induced shifts that enriched for antibiotic-producing Actinobacteria. Future investigation will experimentally confirm whether these specific sectors of the citrus microbiome act as keystone or beneficial species and identify those that may be part of a disease-induced microbiome-mediated immune response. Such experimental evidence will lay the foundation for consideration of practices that enrich for or enhance microbial keystone and beneficial taxa as part of routine agriculture cultural practices and disease management schemes.

\section{ACKNOWLEDGMENTS}

We thank M. Irey, Southern Gardens Citrus, U.S. Sugar Corp for helpful discussions on the survivor tree phenotype and identifying orchards with survivor trees; J. Salvatore and J. Brady, United States Department of Agriculture-Agricultural Research Service, Fort Pierce, FL for their the technical assistance; and K. Kelly and N. Maharaj, University of California-Davis for their assistance with sample collection and processing.

\section{LITERATURE CITED}

Allison, S. D., and Martiny, J. B. H. 2008. Resistance, resilience, and redundancy in microbial communities. Proc. Natl. Acad. Sci. U.S.A. 105: $11512-11519$.

Babcock, B. 2018. Economic impact of California's citrus industry. Citrograph 9:36-39.

Baffoni, L., Accorsi, M., Gaggia, F., Bosi, S., Marotti, I., Biavati, B., Di Gioia, D., and Dinelli, G. 2012. Inoculation with "effective microorganisms" of Lolium perenne L.: Evaluation of plant growth parameters and endophytic colonization of roots. Environ. Eng. Manage. J. 11:S144.

Baker, R. A. 1994. Potential dietary benefits of citrus pectin and fiber. Food Technol. 48:133-136, 138-139. https://agris.fao.org/agris-search/search.do? recordID=US9531775

Bent, E., and Chanway, C. P. 1998. The growth-promoting effects of a bacterial endophyte on lodgepole pine are partially inhibited by the presence of other rhizobacteria. Can. J. Microbiol. 44:980-988.

Berthelot, C., Leyval, C., Foulon, J., Chalot, M., and Blaudez, D. 2016. Plant growth promotion, metabolite production and metal tolerance of dark septate endophytes isolated from metal-polluted poplar phytomanagement sites. FEMS Microbiol. Ecol. 92:fiw144.

Bilodeau, G. J., Martin, F. N., Coffey, M. D., and Blomquist, C. L. 2014. Development of a multiplex assay for genus- and species-specific detection of Phytophthora based on differences in mitochondrial gene order. Phytopathology 104:733-748.

Blaustein, R. A., Lorca, G. L., Meyer, J. L., Gonzalez, C. F., and Teplitski, M. 2017. Defining the core citrus leaf- and root-associated microbiota: Factors associated with community structure and implications for managing huanglongbing (citrus greening) disease. Appl. Environ. Microbiol. 83: e00210-17.

Carrión, V. J., Perez-Jaramillo, J., Cordovez, V., Tracanna, V., de Hollander, M., Ruiz-Buck, D., Mendes, L. W., van Ijcken, W. F. J., Gomez-Exposito, R., Elsayed, S. S., Mohanraju, P., Arifah, A., van der Oost, J., Paulson, J. N., Mendes, R., van Wezel, G. P., Medema, M. H., and Raaijmakers, J. M. 2019. Pathogen-induced activation of disease-suppressive functions in the endophytic root microbiome. Science 366:606-612.

da Graça, J. V., Douhan, G. W., Halbert, S. E., Keremane, M. L., Lee, R. F., Vidalakis, G., and Zhao, H. 2016. Huanglongbing: An overview of a complex pathosystem ravaging the world's citrus. J. Integr. Plant Biol. 58: 373-387.
Dagulo, L., Danyluk, M. D., Spann, T. M., Valim, M. F., Goodrich-Schneider, R., Sims, C., and Rouseff, R. 2010. Chemical characterization of orange juice from trees infected with citrus greening (Huanglongbing). J. Food Sci. 75: C199-C207.

Deyett, E., Roper, M. C., Ruegger, P., Yang, J., Borneman, J., and Rolshausen, P. E. 2017. Microbial landscape of the grapevine endosphere in the context of Pierce's disease. Phytobiomes J. 3:138-149.

Deyett, E., and Rolshausen, P. E. 2019. Temporal dynamics of the sap microbiome of grapevine under high Pierce's disease pressure. Front. Plant Sci. 10:1246.

Dimitrijević, S., Pavlović, M., Maksimović, S., Ristić, M., Filipović, V., Antonović, D., and Dimitrijević-Branković, S. 2018. Plant growth-promoting bacteria elevate the nutritional and functional properties of black cumin and flaxseed fixed oil. J. Sci. Food Agric. 98:1584-1590.

Economos, C., and Clay, W. D. 1999. Nutritional and health benefits of citrus fruits. Energy 62:37.

Edgar, R. C. 2013. UPARSE: Highly accurate OTU sequences from microbial amplicon reads. Nat. Methods 10:996-998.

Edgar, R. C., and Flyvberg, H. 2015. Error filtering, pair assembly and error correction for next-generation sequencing reads. Bioinformatics 21 : 3476-3482.

Essarioui, A., LeBlanc, N., Kistler, H. C., and Kinkel, L. L. 2017. Plant community richness mediates inhibitory interactions and resource competition between streptomyces and fusarium populations in the rhizosphere. Microb. Ecol. 74:157-167.

Essarioui, A., LeBlanc, N., Otto-Hanson, L., Schlatter, D. C., Kistler, H. C., and Kinkel, L. L. 2019. Inhibitory and nutrient use phenotypes among coexisting Fusarium and Streptomyces populations suggest local coevolutionary interactions in soil. Environ. Microbiol. 22:976-985.

Folimonova, S. Y., and Achor, D. S. 2010. Early events of citrus greening (Huanglongbing) disease development at the ultrastructural level. Phytopathology 100:949-958.

Fujiwara, K., Iwanami, T., and Fujikawa, T. 2018. Alterations of Candidatus Liberibacter asiaticus-associated microbiota decrease survival of $\mathrm{Ca}$. L. asiaticus in in vitro assays. Front. Microbiol. 9:3089.

Giassi, V., Kiritani, C., and Kupper, K. C. 2016. Bacteria as growth-promoting agents for citrus rootstocks. Microbiol. Res. 190:46-54.

Ginestet, C. 2011. ggplot2: Elegant graphics for data analysis. J. R. Stat. Soc. [Ser A] 174:245-246.

Ginnan, N. A., Dang, T., Bodaghi, S., Ruegger, P. M., Peacock, B. B., McCollum, G., England, G., Vidalakis, G., Roper, C., Rolshausen, P., and Borneman, J. 2018. Bacterial and fungal next generation sequencing datasets and metadata from citrus infected with 'Candidatus Liberibacter asiaticus'. Phytobiomes J. 2:64-70.

Gonzalez, P., Reyes, J., and Etxeberria, E. 2011. Starch analysis of HLB-affected and control healthy citrus leaves reveal variations in the amylose/amylopectin ratio. Proc. Fla. State Hortic. Soc. 124:69-75.

Gottwald, T. R. 2010. Current epidemiological understanding of citrus Huanglongbing. Annu. Rev. Phytopathol. 48:119-139.

Graham, J. H., Johnson, E. G., Gottwald, T. R., and Irey, M. S. 2013. Presymptomatic fibrous root decline in citrus trees caused by huanglongbing and potential interaction with Phytophthora spp. Plant Dis. 97:1195-1199.

Hervé, M. 2015. RVAideMemoire: Diverse Basic Statistical and Graphical Functions. R package version 0.9-45-2. https://cran.microsoft.com/snapshot/ 2017-08-01/web/packages/RVAideMemoire/index.html

Hoegger, P. J., Navarro-González, M., Kilaru, S., Hoffmann, M., Westbrook, E. D., and Kües, U. 2004. The laccase gene family in Coprinopsis cinerea (Coprinus cinereus). Curr. Genet. 45:9-18.

Hussein, K. A., and Joo, J. H. 2018. Plant growth-promoting rhizobacteria improved salinity tolerance of Lactuca sativa and Raphanus sativus. J. Microbiol. Biotechnol. 28:938-945.

Ignatova, L. V., Brazhnikova, Y. V., Berzhanova, R. Z., and Mukasheva, T. D. 2015. Plant growth-promoting and antifungal activity of yeasts from dark chestnut soil. Microbiol. Res. 175:78-83.

Kim, K., Yim, W., Trivedi, P., Madhaiyan, M., Deka Boruah, H. P., Islam, M. R., Lee, G., and Sa, T. 2010. Synergistic effects of inoculating arbuscular mycorrhizal fungi and Methylobacterium oryzae strains on growth and nutrient uptake of red pepper (Capsicum annuиm L.). Plant Soil 327: 429-440.

Klein, M. N., and Kupper, K. C. 2018. Biofilm production by Aureobasidium pullulans improves biocontrol against sour rot in citrus. Food Microbiol. 69: 1-10.

Lahti, L., Shetty, S., Blake, T., and Salojarvi, J. 2017. Microbiome R package. https://github.com/microbiome/microbiome 
Lamichhane, J. R., and Venturi, V. 2015. Synergisms between microbial pathogens in plant disease complexes: A growing trend. Front. Plant Sci. 6: 385.

Lavermicocca, P., Valerio, F., and Visconti, A. 2003. Antifungal activity of phenyllactic acid against molds isolated from bakery products. Appl. Environ. Microbiol. 69:634-640.

Lazarotto, M., Muniz, M. F. B., Dos Santos, R. F., Blume, E., Harakawa, R., and Hamann, F. A. 2014. First report of Fusarium equiseti associated on pecan (Carya illinoinensis) seeds in Brazil. Plant Dis. 98:847.

Liu, W., Sun, F., and Hu, Y. 2018. Genome mining-mediated discovery of a new avermipeptin analogue in Streptomyces actuosus ATCC 25421. ChemistryOpen 7:558-561.

Lu, N.-H., Huang, Q.-Z., He, H., Li, K.-W., and Zhang, Y.-B. 2014. First report of black stem of Avicennia marina caused by Fusarium equiseti in China. Plant Dis. 98:843.

Madhaiyan, M., Alex, T. H. H., Ngoh, S. T., Prithiviraj, B., and Ji, L. 2015. Leafresiding Methylobacterium species fix nitrogen and promote biomass and seed production in Jatropha curcas. Biotechnol. Biofuels 8:222.

McMurdie, P. J., and Holmes, S. 2013. phyloseq: An R package for reproducible interactive analysis and graphics of microbiome census data. PLoS One 8: e61217.

Mendes, R., Kruijt, M., de Bruijn, I., Dekkers, E., van der Voort, M., Schneider, J. H. M., Piceno, Y. M., DeSantis, T. Z., Andersen, G. L., Bakker, P. A. H. M., and Raaijmakers, J. M. 2011. Deciphering the rhizosphere microbiome for disease-suppressive bacteria. Science 332:1097-1100.

Miles, T. D., Martin, F. N., Robideau, G. P., Bilodeau, G. J., and Coffey, M. D. 2017. Systematic development of Phytophthora species-specific mitochondrial diagnostic markers for economically important members of the genus. Plant Dis. 101:1162-1170.

Müller, D. B., Vogel, C., Bai, Y., and Vorholt, J. A. 2016. The plant microbiota: Systems-level insights and perspectives. Annu. Rev. Genet. 50:211-234.

Oksanen, J., Kindt, R., Legendre, P., O’Hara, B., Stevens, M. H. H., Oksanen, M. J., and Suggests, M. 2007. The vegan package. Community Ecol. Package 10:631-637.

Quattrini, M., Bernardi, C., Stuknyte, M., Masotti, F., Passera, A., Ricci, G., Vallone, L., De Noni, I., Brasca, M., and Fortina, M. G. 2018. Functional characterization of Lactobacillus plantarum ITEM 17215: A potential biocontrol agent of fungi with plant growth promoting traits, able to enhance the nutritional value of cereal products. Food Res. Int. 106:936-944.

Réblová, M., Untereiner, W. A., and Réblová, K. 2013. Novel evolutionary lineages revealed in the Chaetothyriales (fungi) based on multigene phylogenetic analyses and comparison of its secondary structure. PLoS One 8:e63547.

Riera, N., Handique, U., Zhang, Y., Dewdney, M. M., and Wang, N. 2017. Characterization of antimicrobial-producing beneficial bacteria isolated from huanglongbing escape citrus trees. Front. Microbiol. 8:2415.

Ritpitakphong, U., Falquet, L., Vimoltust, A., Berger, A., Métraux, J.-P., and L'Haridon, F. 2016. The microbiome of the leaf surface of Arabidopsis protects against a fungal pathogen. New Phytol. 210:1033-1043.

Rolli, E., Marasco, R., Vigani, G., Ettoumi, B., Mapelli, F., Deangelis, M. L., Gandolfi, C., Casati, E., Previtali, F., Gerbino, R., Pierotti Cei, F., Borin, S., Sorlini, C., Zocchi, G., and Daffonchio, D. 2015. Improved plant resistance to drought is promoted by the root-associated microbiome as a water stressdependent trait. Environ. Microbiol. 17:316-331.

Rouse, R., Roberts, P., Irey, M., Boyd, M., and Willis, T. 2010. Monitoring trees infected with huanglongbing in a commercial grove receiving nutritional/SAR foliar sprays in southwest Florida. Proc. Fla. State Hortic. Soc. 123:118-120.

Ruegger, P. M., Clark, R. T., Weger, J. R., Braun, J., and Borneman, J. 2014. Improved resolution of bacteria by high throughput sequence analysis of the rRNA internal transcribed spacer. J. Microbiol. Methods 105:82-87.

Sagaram, U. S., DeAngelis, K. M., Trivedi, P., Andersen, G. L., Lu, S.-E., and Wang, N. 2009. Bacterial diversity analysis of huanglongbing pathogeninfected citrus, using PhyloChip arrays and 16S rRNA gene clone library sequencing. Appl. Environ. Microbiol. 75:1566-1574.
Schanche, M., Avershina, E., Dotterud, C., Øien, T., Storrø, O., Johnsen, R., and Rudi, K. 2015. High-resolution analyses of overlap in the microbiota between mothers and their children. Curr. Microbiol. 71:283-290.

Schlaeppi, K., and Bulgarelli, D. 2015. The plant microbiome at work. Mol. Plant-Microbe Interact.28:212-217.

Tani, A., Sahin, N., Fujitani, Y., Kato, A., Sato, K., and Kimbara, K. 2015. Methylobacterium Species promoting rice and barley growth and interaction specificity revealed with whole-cell matrix-assisted laser desorption/ ionization-time-of-flight mass spectrometry (MALDI-TOF/MS) analysis. PLoS One 10:e0129509.

Timmer, L. W., Garnsey, S. M., and Graham, J. H. 1999. Compendium of Citrus Diseases, 2nd ed. American Phytopathological Society, St. Paul, MN, U.S.A.

Trivedi, P., Duan, Y., and Wang, N. 2010. Huanglongbing, a systemic disease, restructures the bacterial community associated with citrus roots. Appl. Environ. Microbiol. 76:3427-3436.

Trivedi, P., Trivedi, C., Grinyer, J., Anderson, I. C., and Singh, B. K. 2016. Harnessing host-vector microbiome for sustainable plant disease management of phloem-limited bacteria. Front. Plant Sci. 7:1423.

Valerio, F., Lavermicocca, P., Pascale, M., and Visconti, A. 2004. Production of phenyllactic acid by lactic acid bacteria: An approach to the selection of strains contributing to food quality and preservation. FEMS Microbiol. Lett. 233:289-295.

Vandenkoornhuyse, P., Quaiser, A., Duhamel, M., Le Van, A., and Dufresne, A. 2015. The importance of the microbiome of the plant holobiont. New Phytol. 206:1196-1206.

Van der Ent, S., Van Hulten, M., Pozo, M. J., Czechowski, T., Udvardi, M. K., Pieterse, C. M. J., and Ton, J. 2009. Priming of plant innate immunity by rhizobacteria and beta-aminobutyric acid: Differences and similarities in regulation. New Phytol. 183:419-431.

Vannier, N., Agler, M., and Hacquard, S. 2019. Microbiota-mediated disease resistance in plants. PLoS Pathog 15:e1007740.

Vorholt, J. A. 2012. Microbial life in the phyllosphere. Nat. Rev. Microbiol. 10: 828-840.

Wang, J.-L., Li, T., Liu, G.-Y., Smith, J. M., and Zhao, Z.-W. 2016. Unraveling the role of dark septate endophyte (DSE) colonizing maize (Zea mays) under cadmium stress: Physiological, cytological and genic aspects. Sci. Rep. 6: 22028.

Wang, N., and Gmitter, F. 2014. What can we learn from HLB survivor trees? Citrus Ind. July:16-17.

Wang, N., Jin, T., Trivedi, P., Setubal, J. C., Tang, J., Machado, M. A., Triplett, E., Coletta-Filho, H. D., Cubero, J., Deng, X., Wang, X., Zhou, C., Ancona, V., Lu, Z., Dutt, M., Borneman, J., Rolshausen, P. E., Roper, C., Vidalakis, G., Capote, N., Catara, V., Pietersen, G., Al-Sadi, A. M., Srivastava, A., Graham, J. H., Leveau, J., Ghimire, S. R., Vernière, C., and Zhang, Y. 2015. Announcement of the International Citrus Microbiome (Phytobiome) Consortium. J. Citrus Pathol. 2:1-2.

Wang, X., Glawe, D. A., Kramer, E., Weller, D., and Okubara, P. A. 2018. Biological control of Botrytis cinerea: Interactions with native vineyard yeasts from Washington State. Phytopathology 108:691-701.

Willis, A., Rodrigues, B. F., and Harris, P. J. C. 2013. The ecology of arbuscular mycorrhizal fungi. Crit. Rev. Plant Sci. 32:1-20.

Xu, J., Zhang, Y., Zhang, P., Trivedi, P., Riera, N., Wang, Y., Liu, X., Fan, G., Tang, J., Coletta-Filho, H. D., Cubero, J., Deng, X., Ancona, V., Lu, Z., Zhong, B., Roper, M. C., Capote, N., Catara, V., Pietersen, G., Vernière, C., Al-Sadi, A. M., Li, L., Yang, F., Xu, X., Wang, J., Yang, H., Jin, T., and Wang, N. 2018. The structure and function of the global citrus rhizosphere microbiome. Nat. Commun. 9:4894.

Yaseen, T., and D'Onghia, A. M. 2012. Fusarium spp. associated to citrus dry root rot: An emerging issue for Mediterranean citriculture. Acta Hortic. 940: 647-655.

Zhang, Y., Xu, J., Riera, N., Jin, T., Li, J., and Wang, N. 2017. Huanglongbing impairs the rhizosphere-to-rhizoplane enrichment process of the citrus rootassociated microbiome. Microbiome 5:97. 